\title{
Research Paper \\ Prevalence of Fall and Its Related Factors Among Older People in Bojnurd in 2015
}

\author{
Seyed Hamid Nabavi ${ }^{1}$, Seyedeh Tayebeh Hatami ${ }^{2}$, Fatemeh Norouzi ${ }^{3}$, Zakieh Gerivani ${ }^{3}$, Seyed Esmaeil Hatami ${ }^{4},{ }^{*}$ Hadiseh \\ Monadi Ziarat ${ }^{4}$, Ahmad Delbari ${ }^{5}$
}

1. Gerontological Care Research Center, North Khorasan University of Medical Sciences, Bojnurd, Iran.

2. Department of Environmental Health Engineering, School of Public Health, Hamadan University of Medical Sciences, Hamadan, Iran.

3. Department of Public Health, School of Health, North Khorasan University of Medical Sciences, Bojnurd, Iran.

4. Addiction and Behavioral Sciences Research Center, North Khorasan University of Medical Sciences, Bojnurd, Iran.

5. Department of Neurobiology, Karolinska Institute, Stockholm, Sweden.

Received: 11 Feb. 2016

Accepted: 19 Jun. 2016

Key words:

Prevalence, Accidental

fall, Elderly

\section{A B STRACT}

Objectives Falls are a major health problem and a leading cause of injury-related death and disabilities in the elderly. Therefore, the aim of this study was to survey fall prevalence and its related factors among the elderly in Bojnurd.

Methods \& Materials This cross-sectional study was performed on 288 elderly residents in Bojnurd in 2015. By strata sampling, the elderly people were included in the study according to their population in each stratum by randomization. Data was collected by a self-made checklist that included demographic characteristics, history of falls in the last six months, and programs prevent of falls at habitat. Data were analyzed by independent-t and chi-square tests using SPSS 20 software.

Results The fall prevalence rate in elderly people was $30 \%$. In total, $54.2 \%$ of samples were men and $45.8 \%$ were women, and the mean age was $70.42 \pm 8.56$ years. Around $55.1 \%$ falls occurred in home or elderly care center. In this study, there was a significant correlation between fall prevalence and age over 60 years $(\mathrm{P}=0.01)$, consumption of hypnotic and sedative drugs $(P=0.03)$, back pain $(P=0.008)$, arthritis $(P=0.03)$, problems with walking $(P<0.001)$, problems with sitting and standing $(P=0.009)$, hearing problems $(P=0.02)$, environmental factors at home such as status stairs and railings $(P<0.001)$, status cover the floors $(p<0.001)$, status bathroom $(P=0.01)$, status lavatory $(P<0.001)$, and status lighting $(P=0.02)$.

Conclusion Considering the prevalence of falls in elderly people and inadequate prevention measures at home, fall prevention programs should be considered seriously.

\section{* Corresponding Author:}

Hadiseh Monadi Ziarat, MSc.

Address: Addiction and Behavioral Sciences Research Center, North khorasan University of Medical Sciences, Bojnurd, Iran. Tel: +98 (58) 32742088

E-mail: hadisemonadi67@yahoo.com 


\section{Arqf، شيوع سقوط و عوامل مرتبط با آن در سالمندان شهر بجنورد،}

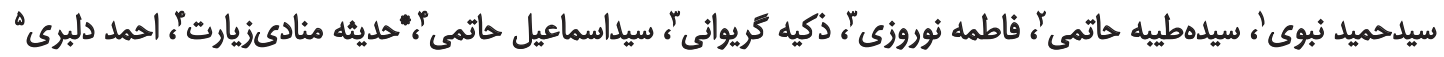

1 - مركز تحقيقات مراقبت سالمئدى، دانشكاه علوم يُششكى خراسانشمالى، بجنورد، ايران.

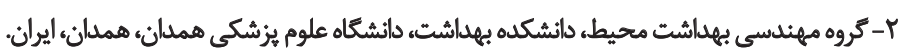

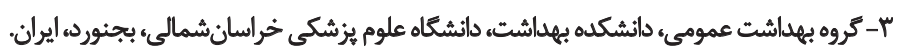

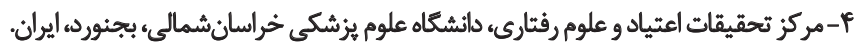

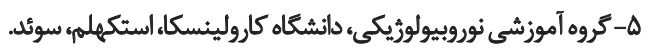

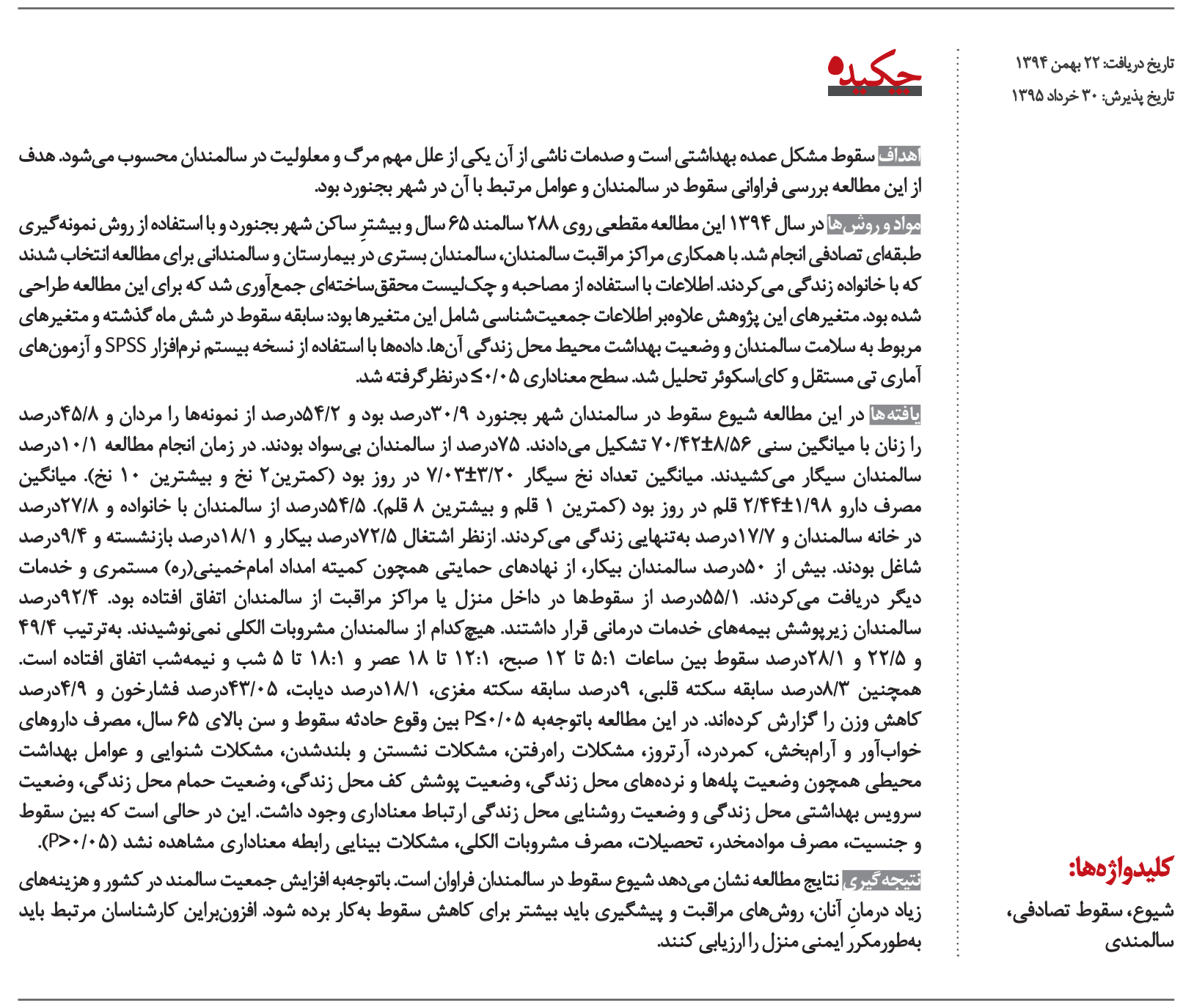

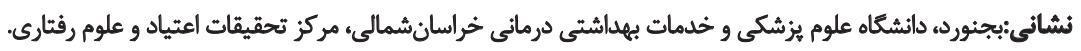

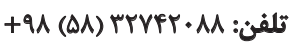
بست الكترونيكي: بلفي 
عوامل متعددى با سقوط در سالمندان مرتبط است. علاوهبر

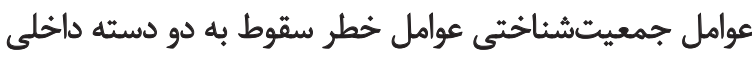

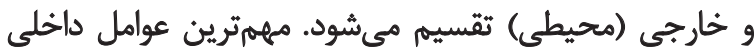

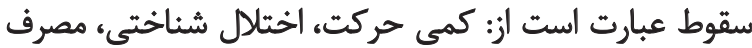

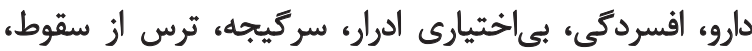

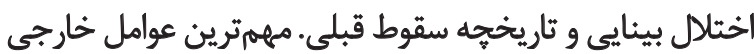

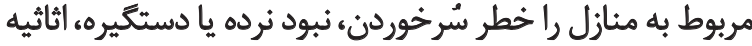

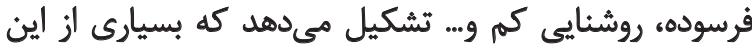

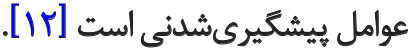

سقوط يكى از شايعترين و جدى ترين مشكلات دوران

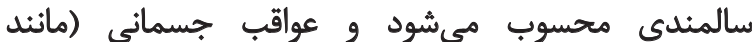

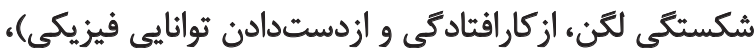

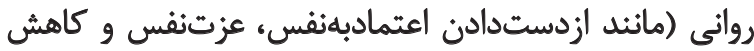

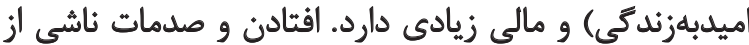

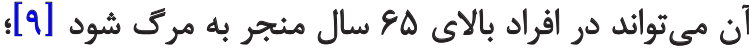

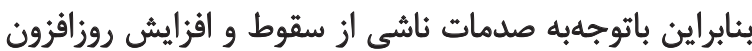

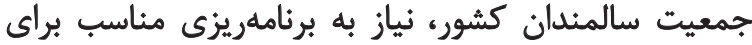

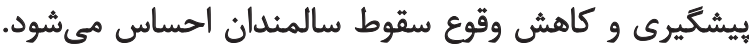

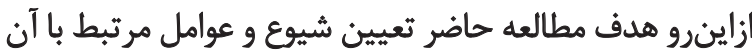

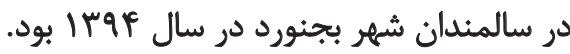

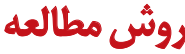

اين مطالعه مقطعى در ساهماهه نخست سال Iraf

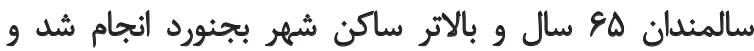

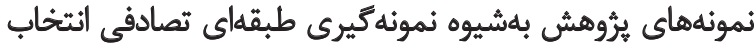

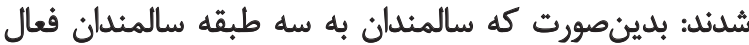

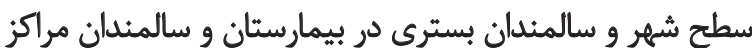

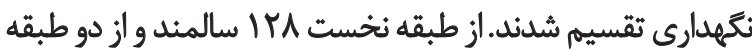

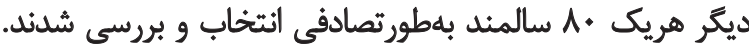

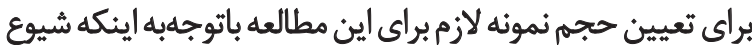

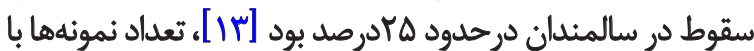

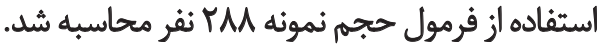

اطلاعات لازم با استفاده از جكليست محققساخته كه شامل

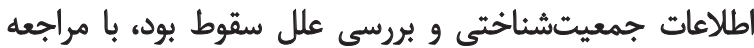

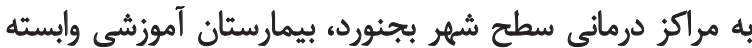

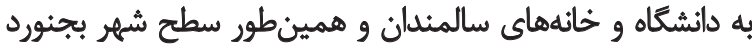

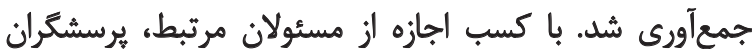

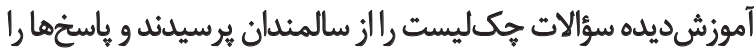

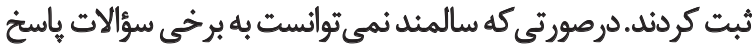

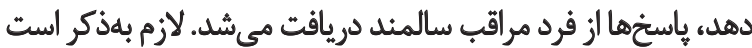

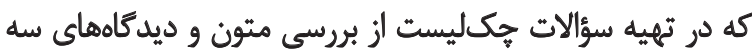
متخصص حوزه سالمندشناسى بهره كرفته شد.

دادهاى حاضر در نسخه بيستم نرم|فزار SPSS وارد و براى
مقلمه

سالمندى دوران حساسى از زندگى بشر است كه طي آن تغييراتى

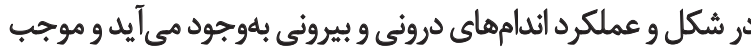

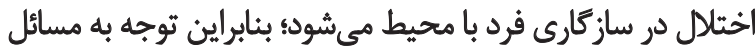

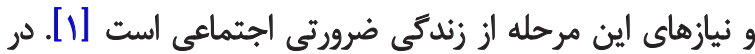

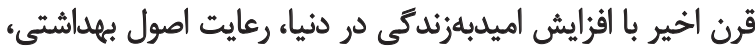

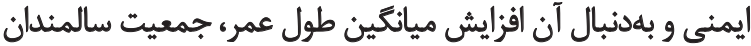

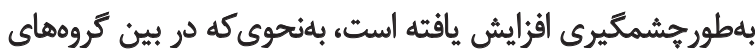

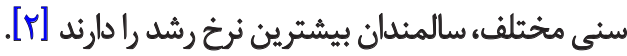
درحالحاضر جمعيت سالمندان جهان داراى رشد

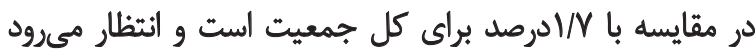

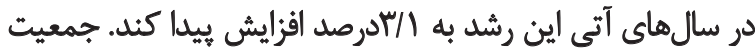

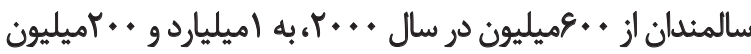

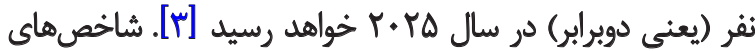

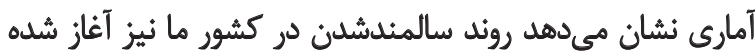

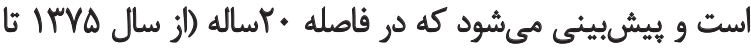
هوبا (1) به ميانه ستّى جمعيت كشور • (1 سال افزوده شود. به كزارش مركز اطلاعات سازمان ملل متحد در تهران، تعداد

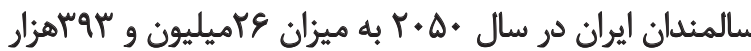

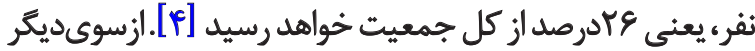

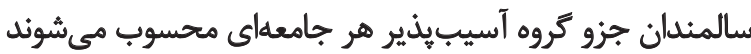

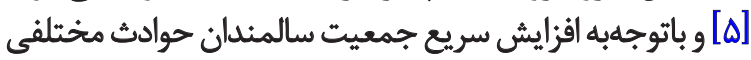
آنها را تهديد مي كند [عائ.

سقوط و آسيبهاى ناشىاز آن يكى از مشكلات شايع سالمندان

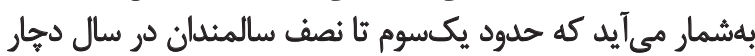

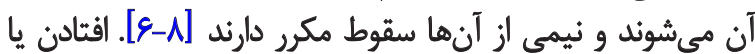

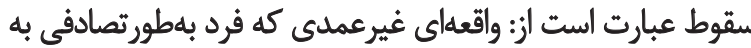

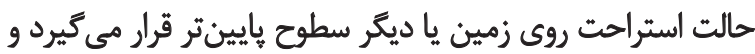

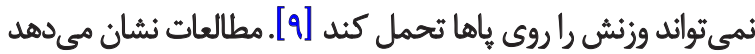
فراواثى سقوط سالمندان در تركيه

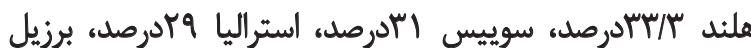
TrV/I

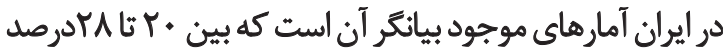

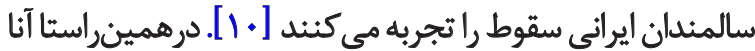

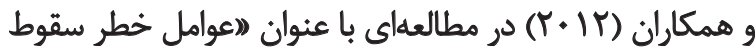

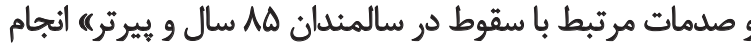

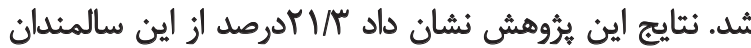

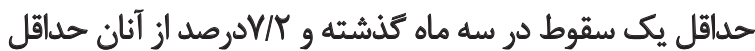

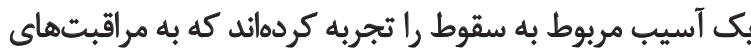

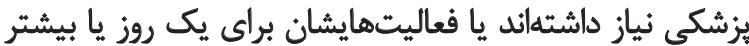

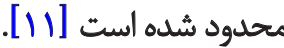


تجزيهوتحليل دادها، از آمار توصيفى (فراوانى و درصد براى يافتهها

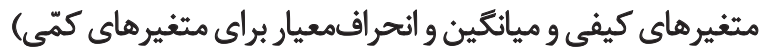

ميزان شيوع سقوط در سالمندان شهر بجنورد، 19 •آدرصد

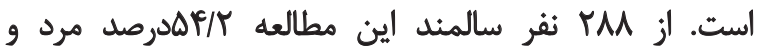

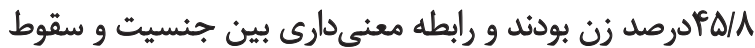
يافت نشد (P=/TA). ميانغين سنى افراد

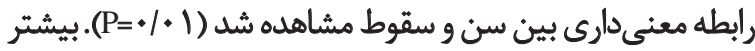

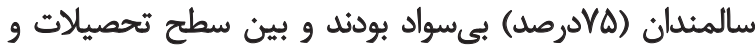

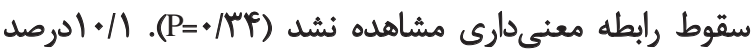

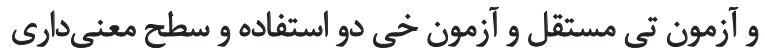

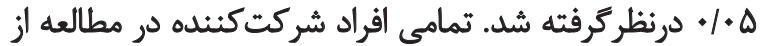

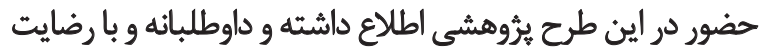

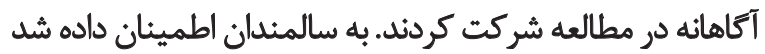

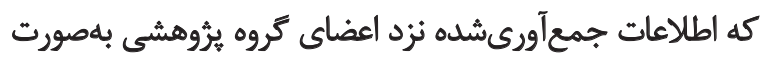

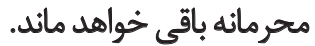

جدول ا. آمار توصيفى مرتبط با سقوط در سالمندان شهر بجنورد در سال Fqجا.

\begin{tabular}{|c|c|c|c|}
\hline درصد & تعداد & \multicolumn{2}{|c|}{ هتغير } \\
\hline$\Delta F / T$ & 108 & مرد | مرد & \multirow{2}{*}{ جنسيت } \\
\hline$P \Delta / \Lambda$ & rir & زن & \\
\hline Va & ME & بيىسواد & \multirow{5}{*}{ تحصيلات } \\
\hline$|N|$ & $\Delta r$ & ابتلاييى & \\
\hline$r / 1$ & 9 & راهنمايع & \\
\hline$r / \mathbb{F}$ & v & دبيرستان & \\
\hline $1 / 9$ & i & مانشكاهى & \\
\hline$r V / A$ & 1.9 & بيكار زيريوشش نهادهاي حمايتى & \multirow{4}{*}{ 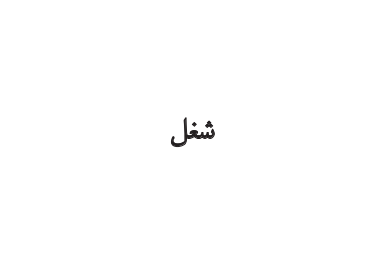 } \\
\hline $\operatorname{men}$ & $1 .$. & بيكار بلونيوشش نهادهاى حمايتى & \\
\hline a/p & rr & شغيل آزاد & \\
\hline WI & $\Delta T$ & بازئشسته & \\
\hline TV/A & ᄉ. & خانه سالمندان & \multirow{3}{*}{ محل زندمى سالمند } \\
\hline$I V / N$ & ه) & تنهايع & \\
\hline$\Delta F / \Delta$ & IQV & همراه خانواده & \\
\hline$r+/ q$ & 19 & 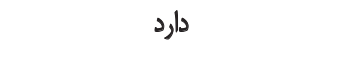 & \multirow{2}{*}{ سابقه حداقل يكبار سقوط نر شش هاه } \\
\hline sal & 199 & ندارد & \\
\hline$\Delta \Delta / 1$ & pq & داخل مثزل يا مركز & \multirow{2}{*}{ محل سقوط } \\
\hline$p e / q$ & p. & خاج هنزل يا مركز & \\
\hline pque & pr & صبح (//ه تا ז1) & \multirow{3}{*}{ زمان سقوط } \\
\hline$r / \Delta$ & r. & 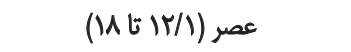 & \\
\hline YN & ro & شب (الN| تاه) & \\
\hline $9 \Upsilon / \%$ & reg & دارد & \multirow{2}{*}{ دفترجه بيمه خذمات درمانى } \\
\hline$V / \varepsilon$ & r & نلدارد & \\
\hline
\end{tabular}


جدول r. ارتباط بين سقوط و وضعيت سلامت سالمندان.

\begin{tabular}{|c|c|c|c|c|c|c|}
\hline \multirow{2}{*}{ P-value } & \multirow{2}{*}{ نسبت شانس (قاصله } & \multirow{2}{*}{ تعداد (TMA) } & \multicolumn{2}{|c|}{ سقوط } & & \multirow{2}{*}{ مثغير } \\
\hline & & & 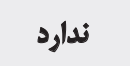 & دارد & & \\
\hline \multirow{2}{*}{.$/ 199$} & \multirow{2}{*}{$V \cdot r(\cdot / E Y-V / Y \cdot)$} & $|m|(F \Delta \%)$ & $9 \cdot(89 \%)$ & $P(M) \%$ & دارد & \multirow{2}{*}{ مصرف موادمخلر } \\
\hline & & $\mid \Delta \vee(\Delta \Delta \%)$ & $1.9(99 \%)$ & PA(MI\%) & ن ت ندارد & \\
\hline \multirow{2}{*}{1} & \multirow{2}{*}{$1(1-1)$} & $\cdot(\cdot \%)$ & $\cdot(\cdot \%)$ & $\cdot(\cdot \%)$ & دارد & \multirow{2}{*}{ مصرف مشروبات الكلى } \\
\hline & & $r M(1 \ldots \%)$ & $199(99 \%)$ & А१(พั\%) & 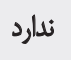 & \\
\hline \multirow{2}{*}{$.1 \cdot r$} & \multirow{2}{*}{$\cdot / \Delta T(\cdot / T A-\cdot / Q S)$} & Vo(re\%) & $\Delta q(v 9 \%)$ & $\mid E(r) \%)$ & 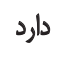 & \multirow{2}{*}{ مصرف داروهاى خوابآور } \\
\hline & & rir $(n+\%)$ & $1 f+(89 \%)$ & $v(\pi+\%)$ & ن نلمارد & \\
\hline \multirow{2}{*}{$\bullet \cdot \cdot 1$} & \multirow{2}{*}{$V / Q V(M / M A-r / T V)$} & $119(\% 1 \%)$ & $n(81 \%)$ & $p v(r q \%)$ & دارد ل & \multirow{2}{*}{ كمردرد } \\
\hline & & $\operatorname{leq}(\Delta 9 \%)$ & $\operatorname{IrV}(V \Delta \%)$ & $r T(T \Delta \%)$ & 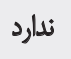 & \\
\hline \multirow{2}{*}{$.1+r$} & \multirow{2}{*}{$r / W(Y / F-\varphi / T E)$} & $W(1 \% \%)$ & $r+(\Delta r \%)$ & $\operatorname{IV}(* \varepsilon \%)$ & 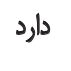 & \multirow{2}{*}{ آ آرتروز } \\
\hline & & raI $\left(A V_{\%}\right)$ & $\ln (Y) \%)$ & $v(r q \%)$ & 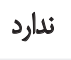 & \\
\hline \multirow{2}{*}{$<+1 .+1$} & \multirow{2}{*}{$r / \Delta Q(Y / \Delta f-r / T \Delta)$} & IFA(FA\%) & A) $(\Delta 9 \%)$ & $\Delta V(F) \%)$ & دارد & \multirow{2}{*}{ مشكل راهرقتن } \\
\hline & & $10 \cdot(\Delta r \%)$ & $111(29 \%)$ & $M(T) \%)$ & 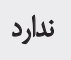 & \\
\hline \multirow{2}{*}{$.10+9$} & \multirow{2}{*}{$\left.V / q \vee\left(M / M A-r /{ }^{\prime}\right)\right)$} & $1.1(10 \%)$ & $8 \cdot(09 \%)$ & $P(p) \%$ & دارد & \multirow{2}{*}{ مشكل نشستن و برخاستن } \\
\hline & & $\operatorname{MV}(8 \Delta \%)$ & $14 q\left(M^{6} \%\right)$ & PA(TE\%) & ن نلارد & \\
\hline \multirow{2}{*}{.$/ \cdot r$} & \multirow{2}{*}{$V / A r(Y / V-r / M)$} & $\Delta V(r \circ \%)$ & $\operatorname{Qr}(\varepsilon \cdot \%)$ & $r \Delta(\varphi \cdot \%)$ & دارد & \multirow{2}{*}{ مشكلات شنوايى } \\
\hline & & $r+1(r \cdot \%)$ & $\operatorname{IfV}\left(\mathrm{N}^{2} \%\right)$ & $\Delta P(T V \%)$ & 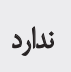 & \\
\hline \multirow{2}{*}{$\cdot / \Delta r$} & \multirow{2}{*}{$1 / M V(\cdot / M-1 / 94)$} & $\| T A(N+\%)$ & $N \in(E \vee \%)$ & $\operatorname{Tr}\left(\pi T^{2} \%\right)$ & دارد & \multirow{2}{*}{ هشكلات بينايي } \\
\hline & & $18 \cdot(\Delta \& \%)$ & $\| r(v)$ & $\operatorname{pr}(r q \%)$ & ن ن ندارد & \\
\hline
\end{tabular}

L

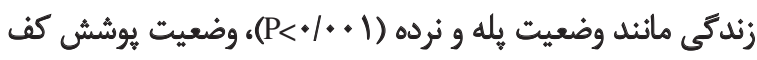

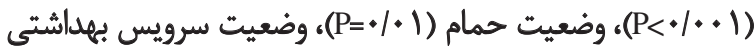

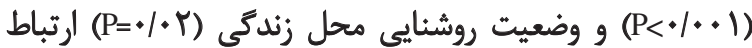
معنى دارى بيدا شد (جدول شماره س). بحث

در اين مطالعه، فراوانى سقوط سالمندان در شهر بجنورة بردان

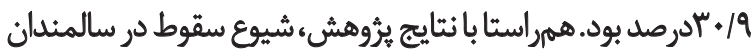

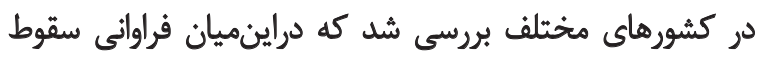
در تركيه

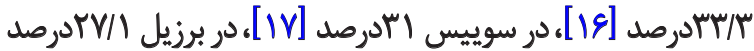

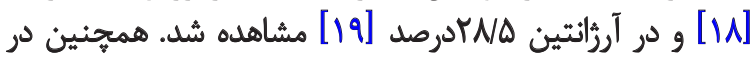

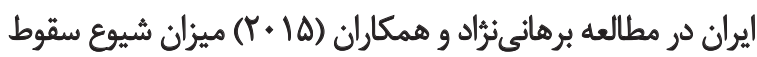

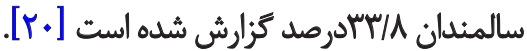

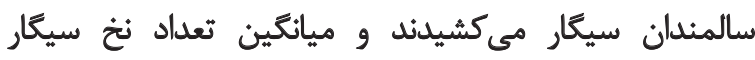

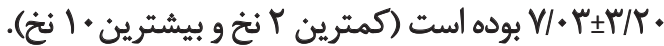
ميانكين مصرف دارو

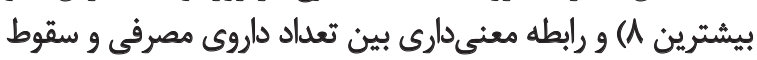

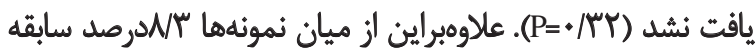

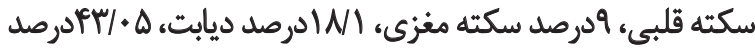

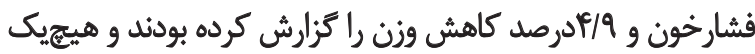

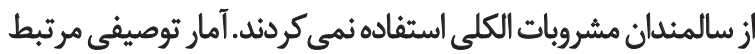
با سقوط در سالمندان در جدول شماره انشان دادئ المدان شده است.

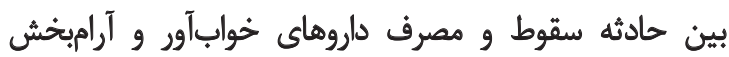

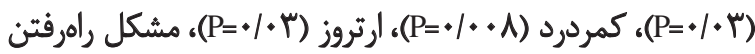

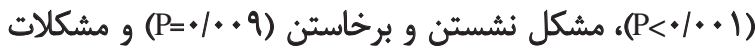

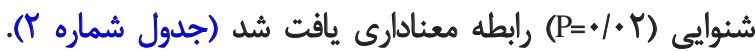

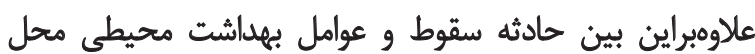


جدول r. ارتباط بين سقوط و وضعيت بهداشت محيط محل زندكى سالمندان.

\begin{tabular}{|c|c|c|c|c|c|c|}
\hline \multirow{2}{*}{ P-value } & \multirow{2}{*}{ نسبت شانس (فاصله } & \multirow{2}{*}{ تعد|د (TMA) } & \multicolumn{2}{|c|}{ سقوط } & & \multirow{2}{*}{ متغير } \\
\hline & & & 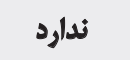 & دارد & & \\
\hline \multirow{2}{*}{$<+1 .+1$} & \multirow{2}{*}{.$/ r E(\cdot / / P-\cdot / P q)$} & & & & & \multirow{2}{*}{ وضعيت زيله وثرده محل } \\
\hline & & $\Delta F(19 \%)$ & $\left.T \Delta(\mu)_{0} \%\right)$ & $r(\Delta \Delta \%)$ & مثاهب نيست & \\
\hline \multirow{2}{*}{$<+1++1$} & \multirow{2}{*}{$\cdot / M F(+/ 1 \cdot-+/ \Delta \Delta)$} & & & $V^{\prime}(Y \wedge \%)$ & & \multirow{2}{*}{ وضميت يوشش كف زفى } \\
\hline & & $r \&(9 \%)$ & $1 \cdot(r A \%)$ & $18(8 \% \%)$ & مناسب نيست & \\
\hline \multirow{2}{*}{.1 .1} & \multirow{2}{*}{$\cdot / F T(\cdot / T \cdot-\cdot / N G)$} & $\operatorname{rar}\left(\mathrm{A}_{\%} \%\right)$ & $M I N T \%)$ & $V(r A \%)$ & منابسب أست & \multirow{2}{*}{ وضعيت حمام هحل } \\
\hline & & $r \Delta(1 Y \%)$ & $W(\Delta) \%)$ & $\operatorname{lv}(F q \%)$ & مناسب نيست & \\
\hline \multirow{2}{*}{$<+1 .+1$} & \multirow{2}{*}{$\cdot / H \cdot(\cdot / N-\cdot / R T)$} & $\operatorname{raf}(M \%)$ & uF(Mr\%) & $V \cdot(T \Lambda \%)$ & مناسب است & \multirow{2}{*}{ 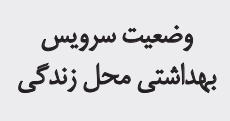 } \\
\hline & & $M F(1 r \%)$ & $1 Q(F F \%)$ & $19(\Delta \% \%)$ & مناسب نيست & \\
\hline \multirow{2}{*}{$.1+r$} & \multirow{2}{*}{.$/ \pi V(\cdot / 10-. / 91)$} & $\operatorname{rev}(9 T \%)$ & Ma(N) & $v \wedge(r q \%)$ & مناسب است & \multirow{2}{*}{ وضعيت روشنايي مهل } \\
\hline & & $r\left(V_{\%} \%\right)$ & $1 .(\% A \%)$ & $M(\Delta r \%)$ & مثاسب نيست & \\
\hline
\end{tabular}

\section{$\therefore$}

يافته ديكًر اين يُروهش نبود ارتباط معنى دار آمارى ميان سطح

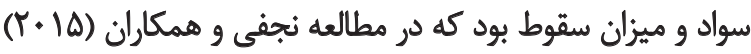

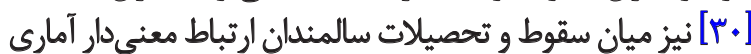

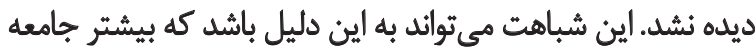

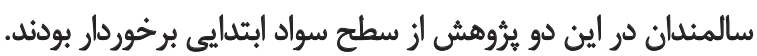

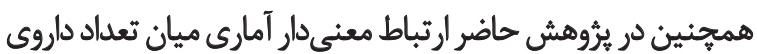

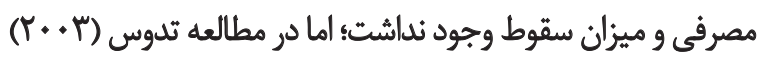

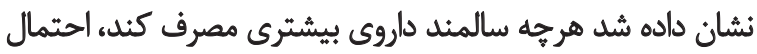

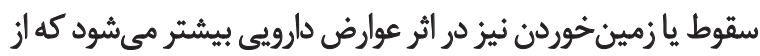
اين نظر با نتيجه مطالعه حاضر تفاوت داشت إسب].

در ئروهش حاضر دربين عوامل مربوط به وضعيت جسمى،

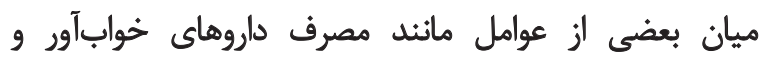

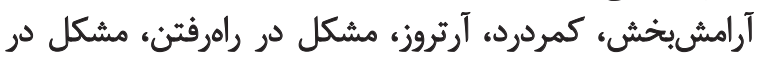

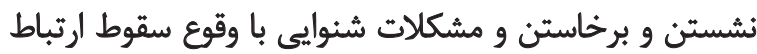

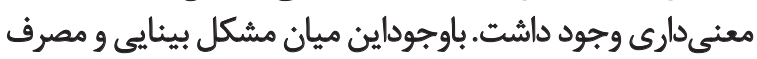

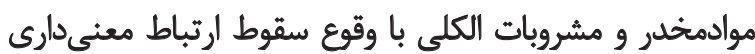

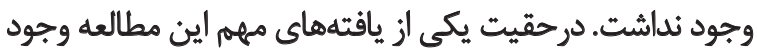

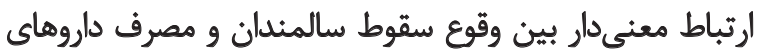

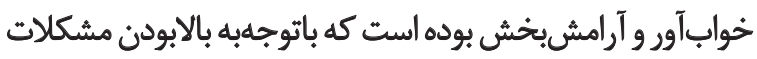

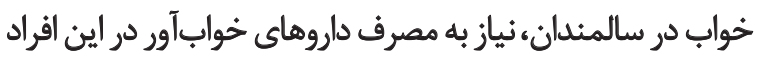

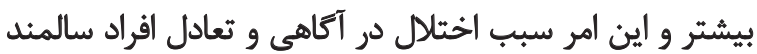

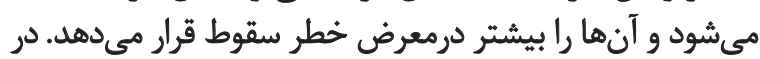

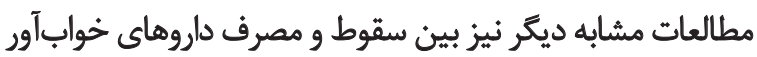

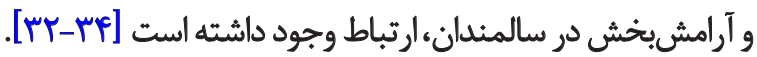

در گزارش سازمان جهاني بهداشت جنسيت بهعنوان عامل خطر

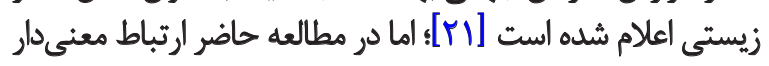

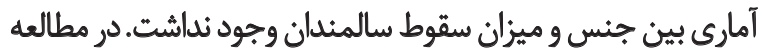

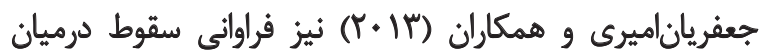

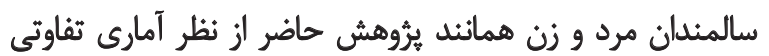

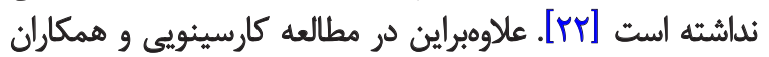

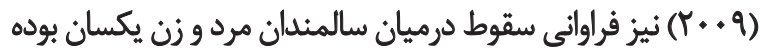

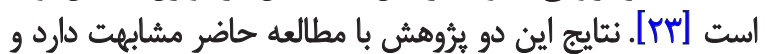

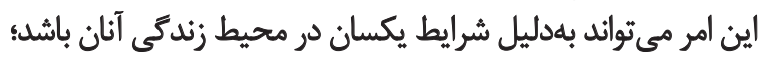

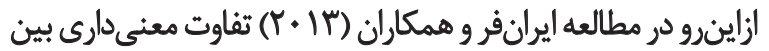

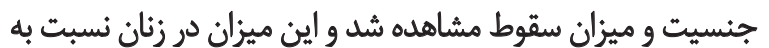

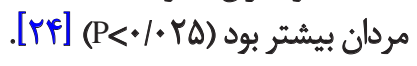

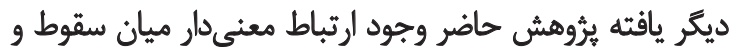

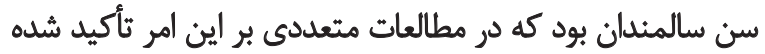

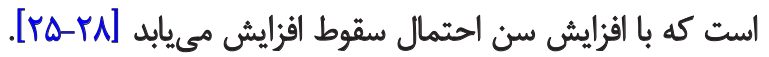

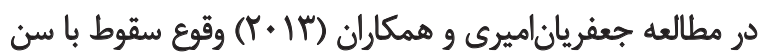

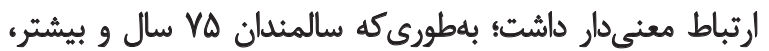

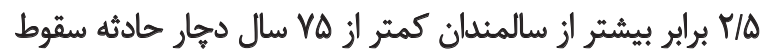

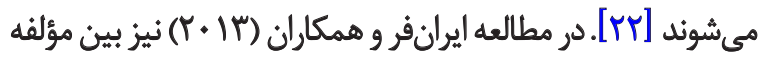

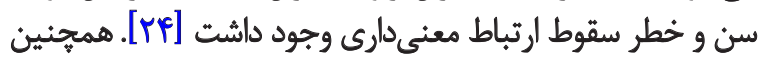

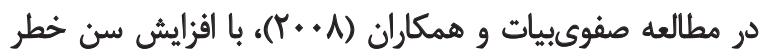

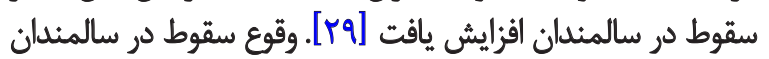

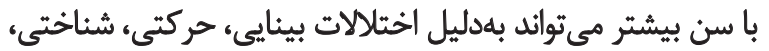

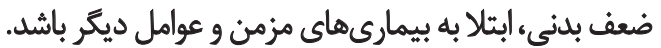




\section{References}

[1] Khademi MJ, Rashedi V, Sajadi S, Moradi Gheshlaghi SH. Anxiety and loneliness in the Iranian older adults. International Journal of Psychology and Behavioral Sciences. 2015; 5(2):49-52. doi: 10.5923/j.ijpbs.20150502.01

[2] Mortazavi S, Eftekhari Ardebili H, Eshaghi R, Dorali R, Shah Siah M, Batlani S. [Effect of continuous body movement at the geriatric psychological health of Shahrekord (Persian)]. Isfahan Medicine Journal. 2010; 29(161):1519-528.

[3] Asadi Noghabi AA, Alhani F, Peyrovi H. [Health in geriatric: a review study (Persian)]. Iran Journal of Nursing. 2011; 25(78):6271.

[4] Nejati V. [Relation between the active memory and vocal psychology in geriatric (Persian)]. Journal of Research in Rehabilitation Sciences. 2012; 8(3):412-17.

[5] Gnanadesigan N, Saliba D, Roth CP, Solomon DH, Chang JT, Schnelle J, et al. The quality of care provided to vulnerable older community-based patients with urinary incontinence. Journal of the American Medical Directors Association. 2004; 5(3):141-46. doi: 10.1016/s1525-8610(04)70106-1

[6] Acimis NM, Mas N, Yazei AC, Gocmen L, Isik T, Mas MR. Accidents of the elderly living in Kocaeli region, Turkey. Archives of Gerontology \& Geriatrics. 2009; 49(20):220-23. doi: 10.1016/j. archger.2008.08.015

[7] Murphy TE, Baker DI, Leo-Summers LS, Allore HG, Tinetti ME. Association between treatmentor usual care region and hospitalization for fall-related traumatic brain injury in the connecticut collaboration for fall prevention. Journal of the American Geriatrics Society. 2013; 61(10):1763-767. doi: 10.1111/jgs.12462

[8] Pedroso RV, Coelho FG, Santos-Galduroz RF, Costa JL, Gobbi $\mathrm{S}$, Stella F. Balance, executive functions and falls in elderly with Alzheimer's disease (AD): a longitudinal study. Archives of Gerontology and Geriatrics. 2012; 54(2):348-51. doi: 10.1016/j.archger.2011.05.029

[9] Maghfouri B, Hassani Mehraban A, Taghizade G, Aminian G, Jafari H. [Validity and reliability of Persion version of home falls and accident screening tool in Iranian elderly (Persian)]. Journal of Modern Rehabilitation. 2012; 5(4):9-14

[10] Jafarian Amiri S, Zabihi A, Aziznejad Roshan P, Hosseini S, Bijani A. [Fall at home and its related factors among the elderly in Babol city, Iran (Persian)]. Journal of Babol University of Medical Sciences. 2013; 15(5):95-110.

[11] Grundstrom AC, Guse CE, Layde PM. Risk factors for falls and fall-related injuries in adults 85 tears of age and older. Archives of Gerontology \& Geriatrics. 2012; 54(3):421-28. doi 10.1016/j.archger.2011.06.008

[12] Sheikh S, Akbari Kamrani A, Karimlo M, Fadayevatan R. [Comparison between the risky agents correspond to fall in elderly people of urban and rural regions of Zabol (Persian)]. Iranian Journal of Ageing. 2013; 8(3):44-52.

[13] Gates S, Fisher JD, Cooke MW, Carter YH, Lamb SE. Multifactorial assessment and targeted intervention for preventing falls and injuries among older people in community and emergency care settings. British Medical Journal. 2008; 336(7636):130-33. doi: 10.1136/bmj.39412.525243.be

[14] Halil M, Ulger Z, Cankurtaran M, Shorbagi A, Yavuz BB, Dede $\mathrm{D}$, et al. Falls and the elderly: Is there any difference in the de-

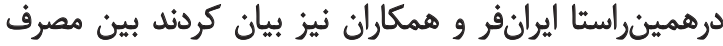

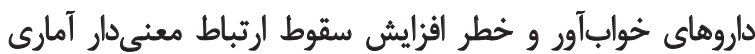

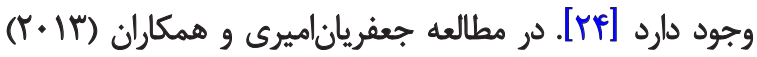

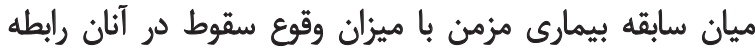

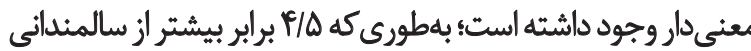

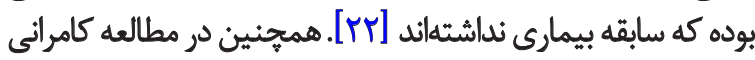

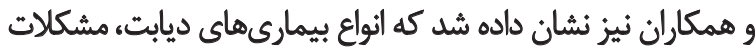

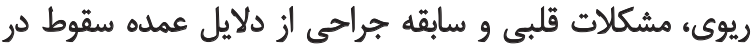

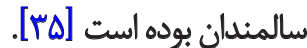

از ديكر يافتههاى مهم اين يثروهش وجود ارتباط معنى دار ميان

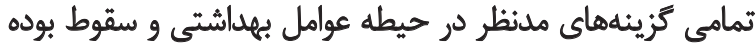

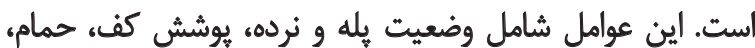

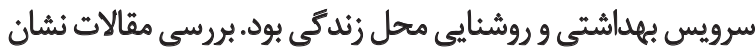

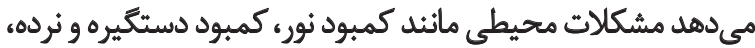

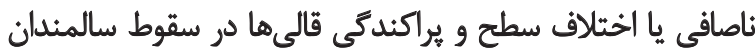

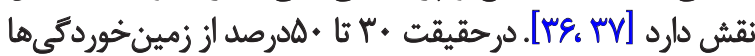

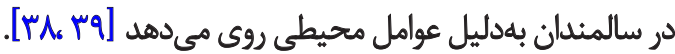

$$
\text { نتيجه كيرى نهايى }
$$

نتايج يُؤوهش حاضر از وجود شيوع بالاي سقوط در سالمندان

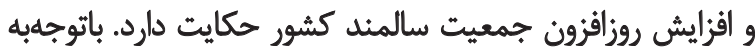

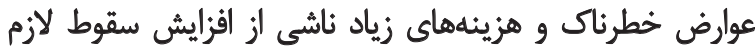

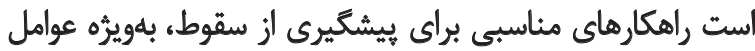

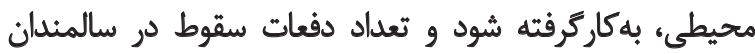

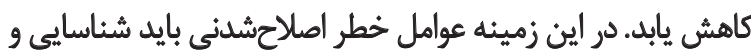

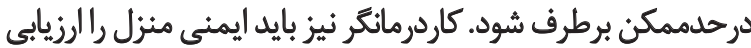

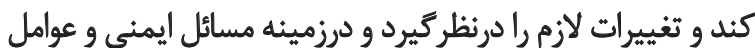

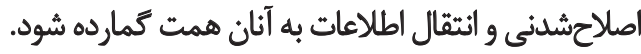

Lugsoso

از محدوديتهاى ثروهش حاضر مى توان به رويكرد مطالعه

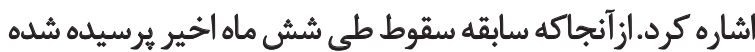

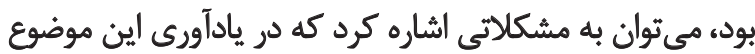

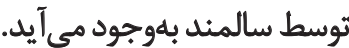

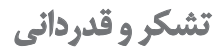

مطالعه حاضر در شوراى ثرؤشش معاونت تحقيقات و

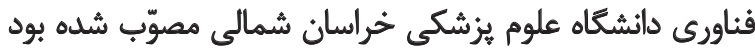

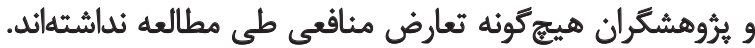

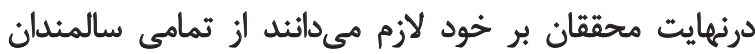
شركت كنئده در اين يثروهش قدردانى لازم كنئد. 
veloping world? a cross-sectional study from Turkey. Archives of Gerontology \& Geriatrics. 2006; 43(3):351-59. doi: 10.1016/j. archger.2005.12.005

[15] Chu LW, Chau AY, Chi I. Falls and subsequent health service utilization in community-dwelling Chinese older adults. Archives of Gerontology \& Geriatrics. 2008; 46(2):125-35. doi 10.1016/j.archger.2007.03.005

[16] Stalenhoef PA, Diederiks JPM, Knottnerus JA, Kester AD, Crebolder HF. A risk model for the prediction of recurrent falls in community-dwelling elderly: a prospective cohort study. Journal of Clinical Epidemiology. 2002; 55(11):1088-094. doi: 10.1016/ s0895-4356(02)00502-4

[17] Swanenburg J, Bruin ED, Uebelhart D, Mulder T. Falls prediction in elderly people: a 1-year prospective study. Gait \& Posture. 2010;31(3):317-21. doi: 10.1016/j.gaitpost.2009.11.013

[18] Morris M, Osborne D, Hill K, Kendig H, Lundgren-Lindquist B, Browning C, et al. Predisposing factors for accasional and multiple falls in older Australians who live at home. Australian Journal of Physiotherapy. 2004; 50(3):153-59. doi: 10.1016/s00049514(14)60153-7

[19] Coimbra AM, Ricci NA, Coimbra IB, Lavras Costallat LT. Falls in the elderly of the family health program. Archives of Gerontology \& Geriatrics. 2010; 51(3):317-22. doi 10.1016/j.archger.2010.01.010

[20] Borhaninejad VR, Rashedi V, Tabe R, Delbari A, Ghasemzadeh $\mathrm{H}$. [Relationship between fear of falling and physical activity in older adults (Persian)]. Medical Journal of Mashhad University of Medical Sciences. 2015; 58(8):446-52.

[21] Yoshida S. A global report on falls prevention epidemiology of falls. [Internet]. 2007 [Cited 2007 Mar 7]. Available from: http:// www.who.int/ageing/projects/1.Epidemiology $\% 20$ of $\% 20$ falls\%20in\%20older\%20age.pdf

[22] Jafarian Amiri S, Zabihi A, Aziznejad Roshan P, Hosseini S, Bijani A. [Fall at home and its related factors among the elderly in Babol city, Iran (Persian)]. Journal of Babol University of Medical Sciences. 2013; 15(5):95-101.

[23] Corsinovi L, Bo M, Ricauda Aimonino N, Marinello R, Gariglio F, Marchetto C, et al. Predictors of falls and hospitalization outcomes in elderly patients admitted to an acute geriatric unit. Archives of Gerontology \& Geriatrics. 2009; 49(1):142-45.

[24] Iranfar M, Ainy E, Soori H. [Fall epidemiology in the elderly residents of care centers in Tehran, 2010 (Persian)]. Iranian Journal of Ageing. 2013; 8(2):30-38.

[25] Ungar A, Rafanelli M, IacomelliI, Brunetti MA, Ceccofiglio A, Tesi F, Marchionni N. Fall prevention in the elderly. Clinical Cases in Mineral and Bone Metabolism. 2013; 10(2):91-95.

[26] Laessoe U, Hoeck HC, Simonsen O, Sinkjaer T, Voigt M. Fall risk in an active elderly population. Journal of Negative Results in BioMedicine. 2007; 26:2. doi: 10.1186/1477-5751-6-2

[27] Dionyssiotis Y. Analyzing the problem of falls among older people. International Journal of General Medicine. 2012; 5:805-13. doi: 10.2147/IJGM.S32651

[28] Almeida ST, Soldera CL, Carli GA, Gomes I, Resende TL. Analysis of extrinsic and intrinsic factors that predispose elderly individuals to fall. Revista da Associação Médica Brasileira. 2012 58(4):427-33.
[29] Safavi Bayat Z, Zorriasatain F. [Determining risk factors associated with falling among elderly at residential care facilities in Tehran (Persian)]. Journal of Qazvin University of Medical Sciences. 2008; 11(4):66-70.

[30] Najafi Ghezlcheh T, Ariapour S, Jafari Oori M. [Epidemiology and relationship of falls and fear of falling in elderly at Kamrani nursing home in Tehran (Persian)]. Iranian Journal of Ageing. 2016; 10(4):152-61.

[31] Theodos PH. Fall prevention in frail elderly nursing home residents: a challenge case management. Lippincott's Case Management. 2003; 8(6):246-51.

[32] Cumming RG, Salkeld G, Thomas M, Szonyi G. Prospective study of the impact of fear of falling on activities of daily living, SF-36 scores, and nursing home admission. Journal of Gerontology. 2000; 55(5):299-305. PMID: 10819321

[33] Gillespie SM, Friedman SM. Fear of falling in new long-term care enrollees. Journal of the American Medical Directors Association. 2007; 8(5):307-13. doi 10.1016/j.jamda.2007.04.006

[34] Andersson AG, Kamwendo K, Appelros P. Fear of falling in stroke patients: relationship with previous falls and functional characteristics. International Journal of Rehabilitation Research. 2008; 31(3):261-64. doi: 10.1097/mrr.0b013e3282fba390

[35] Akbari Kamrani AA, Azadi F, Foroughan M, Siadat S. [Features of falls in elderly people living in nursing homes (Persian)]. Iranian Journal of Ageing. 2006; 1(2):101-05.

[36] Lord SR, Sherrington C, Menz HB. Falls in older people: risk factors and strategies for prevention. Cambridge: Cambridge University Press; 2007.

[37] Shimada H, Suzukawa M, Ishizaki T, Kobayashi K, Kim H, Suzuki $\mathrm{T}$. Relationship between subjective fall risk assessment and falls and fall-related fractures in frail elderly people. BMC Geriatrics. 2011; 11:40. doi: 10.1186/1471-2318-11-40

[38] Axer H, Axer M, Sauer H, Witte OW, Hagemann G. Falls and gait disorders in geriatric neurology. Clinical Neurology and Neurosurgery. 2010; 112(4):265-74. doi 10.1016/j.clineuro.2009.12.015

[39] Grundstrom AC, Guse CE, Layde PM. Risk factors for falls and fall-related injuries in adults 85 years of age and older. Archives of Gerontology and Geriatrics. 2012; 54(3):421-28. doi: 10.1016/j. archger.2011.06.008 